2-1995

\title{
Closed Orbit Bifurcations in Continuum Stark Spectra
}

Michael Courtney

Hong Jiao

Neal Spellmeyer

Daniel Kleppner

J. Gao

William \& Mary

See next page for additional authors

Follow this and additional works at: https://scholarworks.wm.edu/aspubs

Part of the Physics Commons

\section{Recommended Citation}

Courtney, Michael; Jiao, Hong; Spellmeyer, Neal; Kleppner, Daniel; Gao, J.; and Delos, John B., Closed Orbit Bifurcations in Continuum Stark Spectra (1995). Physical Review Letters, 74(9), 1538-1541.

https://doi.org/10.1103/PhysRevLett.74.1538

This Article is brought to you for free and open access by the Arts and Sciences at W\&M ScholarWorks. It has been accepted for inclusion in Arts \& Sciences Articles by an authorized administrator of W\&M ScholarWorks. For more information, please contact scholarworks@wm.edu. 
Authors

Michael Courtney, Hong Jiao, Neal Spellmeyer, Daniel Kleppner, J. Gao, and John B. Delos 


\title{
Closed Orbit Bifurcations in Continuum Stark Spectra
}

\author{
Michael Courtney, Hong Jiao, Neal Spellmeyer, and Daniel Kleppner \\ Department of Physics and Research Laboratory of Electronics, Massachusetts Institute of Technology, 77 Massachusetts Avenue, \\ Cambridge, Massachusetts 02139 \\ J. Gao and J. B. Delos \\ Department of Physics, College of William and Mary, Williamsburg, Virginia 23187
}

(Received 14 August 1994)

\begin{abstract}
We report an experimental and theoretical study of the effect of bifurcations of closed classical orbits in continuum Stark spectra. Our findings provide a natural way to understand how the spectrum evolves from a simple sinusoidal modulation at positive energy to a quasidiscrete spectrum below the classical ionization limit. The experiment employs scaled-energy spectroscopy of lithium. The results provide the first experimental confirmation of a formulation of closed-orbit theory that provides a quantitative description of the behavior of the spectrum at a bifurcation.
\end{abstract}

PACS numbers: $32.60 .+\mathrm{i}, 03.65 . \mathrm{Sq}, 32.80 . \mathrm{Fb}$

The photoionization spectrum of a single-electron atom in an electric field $F$ changes strikingly as the energy decreases [1]. In the positive energy regime (above the zero-field ionization limit), the spectrum is smooth and displays a single periodic oscillation. Below the classical ionization threshold $\left(E_{i}=-2 F^{1 / 2}\right.$, atomic units), the spectrum is quasidiscrete. These contrasting patterns reflect complementary aspects of atomic behavior: the former can be simply interpreted in terms of classical dynamics, while the latter is naturally described in terms of the system's eigenstates. As the energy decreases in the intermediate region from $E>0$ to $E_{i}$, the broad peaks become narrow and new resonances gradually appear. Quantum calculations are possible in this region [2], but they offer little insight into the connections with classical dynamics. However, the region is fertile territory for investigating the connections between quantum and classical descriptions. We report here the results of theoretical and experimental investigations that provide a detailed physical picture of how the system evolves from one extreme to the other. The results are helpful in understanding the connections between quantum and classical behavior in both regular and chaotic systems. They demonstrate the successful repair of formulas which previously diverged at a bifurcation.

Closed-orbit theory $[3,4]$ is a variant of periodic-orbit theory [5] that is well suited to spectroscopy. It provides both an intuitive picture and a quantitative description of how the spectrum evolves based on the behavior of classical orbits that are closed at the nucleus. In the time domain, a quantum wave packet is emitted from the atom and later returns, a process known as a recurrence [6]. Each recurrence gives rise to a sinusoidal modulation of the absorption spectrum. Gao and Delos [4] recently examined closed orbits and recurrences for single-electron atoms in electric fields. At high energy only one orbit exists, accounting for the sinusoidal modulation in the spectrum. As the energy is decreased, new orbits are predicted to spring into existence by an orderly sequence of bifurcations. At each bifurcation, a new frequency component is added to the spectrum.

We studied this bifurcation process experimentally for lithium in an electric field using scaled-energy spectroscopy [7]. Similar studies have been performed in diamagnetic hydrogen [8]. The electric field problem for hydrogen is separable, facilitating a detailed quantitative study of the bifurcation process and the evolution of the spectrum. Alkali-metal atoms in an electric field are fundamentally different from hydrogen because they have nonseparable Hamiltonians and display irregular motion. However, the recurrence spectra are nearly identical for the short-period orbits studied here [7]. Consequently, the lithium spectra can be interpreted in terms of the classical dynamics of hydrogen. At longer periods, the recurrence spectra of alkali-metal atoms can differ significantly from that of hydrogen [9].

The Hamiltonian for hydrogen in an electric field can be rescaled using the substitutions $r=F^{-1 / 2} \tilde{r}$ and $p=$ $F^{1 / 4} \tilde{p}$. The scaled Hamiltonian $\tilde{H}=F^{-1 / 2} H$ does not explicitly depend on the field, and the classical dynamics depends only on the scaled energy $\epsilon=E F^{-1 / 2}$, not on $E$ and $F$ separately.

According to closed-orbit theory, the photoabsorption cross section is given by a smooth slowly varying background plus an oscillatory sum of the form [4]

$$
D f^{\prime}=\sum_{k} \sum_{n=1}^{\infty} D_{n k} \sin \left(n \tilde{S}_{k} F^{-1 / 4}-\Phi_{n k}\right),
$$

where $k$ runs over all of the primitive closed orbits (orbits which are not repetitions), and $n$ runs over repetitions of the primitive orbits. $\tilde{S}_{k}=S_{k} F^{1 / 4}$ is the scaled action of the first repetition of a closed orbit. $D_{n k}$ is the recurrence amplitude of each closed orbit. It contains information about the stability of the orbit, the initial and final angles of the orbit, and the matrix element of the dipole operator between the initial state and zero-energy Coulomb wave. 
$\Phi_{n k}$ is an additional phase which is computed from the Maslov index and related geometrical considerations. The square of the recurrence amplitude $D_{n k}$ is the recurrence strength. Each closed orbit contributes an oscillatory component to the absorption spectrum. The signature of each closed orbit is a peak in the Fourier transform of spectra taken at constant scaled energy and recorded as a function of $F^{-1 / 4}$.

For $E>0$, the only closed orbit is parallel to the field and extends from the nucleus to the classical turning point. The spectrum contains a fundamental Fourier component from this orbit plus harmonics from its repetitions. The parallel orbit is unstable and none of its neighbors return to the nucleus. Consequently, the amplitude of the harmonics decreases roughly exponentially. Just above $E=0$, many harmonics contribute, and the spectrum resembles a sawtooth. The exponential decay rate increases as $\epsilon$ is increased [4], resulting in a single sinusoid at large positive energy $[1,10]$.

As the energy is lowered below $E=0$, new closed orbits are created by bifurcations of the parallel orbit and its repetitions [4]. Because the system is separable in semiparabolic coordinates $\left[u=(r+z)^{1 / 2}, v=(r-\right.$ $\left.z)^{1 / 2}\right]$, the bound trajectories are quasiperiodic. Periodic orbits occur whenever the ratio of periods of $u$ and $v$ motion, $T_{u} / T_{v}$ is a rational fraction $m / l$. Many of these periodic orbits do not touch the nucleus. However, within each family of periodic orbits with period ratio $\mathrm{m} / \mathrm{l}$, there is one closed orbit.

As explained in Ref. [4], the maximum value of the period ratio $T_{u} / T_{v}$ is zero at $\epsilon=0$ and increases as the scaled energy decreases. A graph of the maximum period ratio as a function of scaled energy is shown in Fig. 1. At a given scaled energy, closed orbits exist for every rational period ratio between zero and the maximum. As the scaled energy is decreased, a bifurcation produces a new closed orbit every time the maximum ratio is a

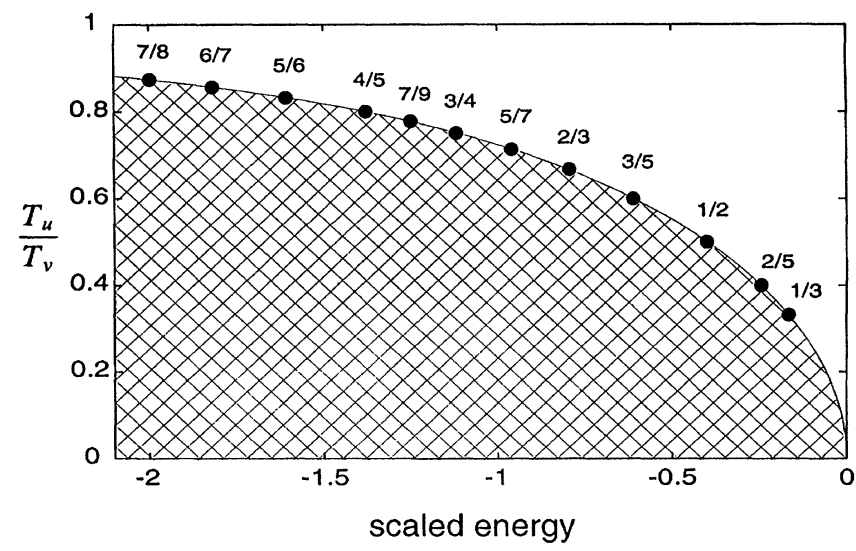

FIG. 1. The maximum period ratio $T_{u} / T_{v}$ as a function of scaled energy. At each scaled energy, all orbits with period ratio in the shaded region are present. Bifurcations occur when this maximum ratio passes through a rational fraction. The short-period bifurcations studied here are indicated. rational number. Short-period orbits correspond to ratios of small integers. Some bifurcations are labeled in Fig. 1.

To illustrate the bifurcation process, pictures of orbits near a bifurcation are shown in Fig. 2. Orbits which are neighbors of a closed orbit (i.e., having the same scaled energy and beginning at the nucleus, but having slightly different initial directions) form a cusp structure upon returning near the nucleus. As the energy is varied, this cusp moves along the $z$ axis: at the bifurcation, the tip of the cusp touches the nucleus. Therefore, near a bifurcation, there is a family of neighbors which also returns to the nucleus. This focusing effect causes a large increase in the recurrence strength as the scaled energy passes through a bifurcation.

In its original formulation, closed-orbit theory diverges at a bifurcation [4,11]. Such divergences are familiar in geometrical optics which predicts infinite intensity if a plane wave is focused to a point. These theories are corrected by accounting for diffraction. Two of the authors (J.G. and J.D.) have modified the closed-orbit theory to include such diffraction effects.

We summarize the features of the theory here. A detailed description will be presented elsewhere. For waves in one dimension the semiclassical formula diverges at an ordinary classical turning point where the behavior of the wave function changes from oscillatory to exponential. This divergence is corrected using Airy's diffraction integral. The same also holds at a simple boundary between classically allowed and forbidden regions in more than one dimension. In two dimensions, it is common for such regions to come together and form a cusp, as in Fig. 2. The associated diffraction integral is known as a Pearcey function [12]. However, the cusp in our problem has a special symmetry: the axis of the cusp coincides with the direction of the electric field, so the cusp is actually three dimensional and cylindrically symmetric about the vertical axis in Fig. 2. Such cusps are produced by an optical lens, and they cause spherical aberration of a point image; the diffraction function that describes them is a Fresnel integral [13].

The cusp in our problem has yet another symmetry. The cusp in Fig. 2 is cylindrically symmetric not only about the vertical axis through the nucleus, but also about (a) outgoing orbits

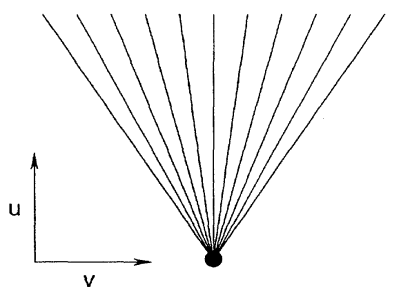

(b) returning orbits

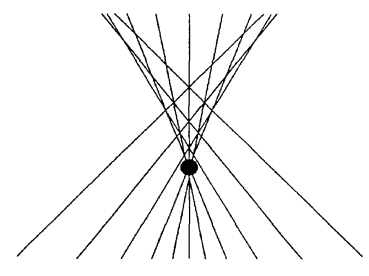

FIG. 2. Cusp structure associated with a family of (a) outgoing and (b) returning orbits near a bifurcation (semiparabolic coordinates). 
the horizontal axis through the nucleus. The formulas that describe this cusp make use of the close connection between a hydrogen atom and a four-dimensional harmonic oscillator. The cusp shown in Fig. 2 is a slice through a structure that exists in a four-dimensional extension of the $(u, v)$ space [14].

In spite of the complexity of the cusp, the formulas for the wave function and recurrence strengths are simple. We find that (i) the recurrence strength is small but nonzero before the classical bifurcation because of wave diffraction effects or tunneling into the classically forbidden region; (ii) as expected, the recurrence strength is finite at the bifurcation; and (iii) the recurrence strength has its maximum after the bifurcation, just as waves are largest slightly inside of a classically allowed boundary.

The structure of bifurcations can be studied experimentally using scaled-energy spectroscopy. The lasers and field are scanned simultaneously so as to keep the scaled energy - and hence the classical dynamicsconstant $[7,8]$. Our experiment employs a lithium atomic beam which passes through holes in the centers of a pair of electric field plates. Between the field plates, one laser excites the $2 S \rightarrow 3 S$ two-photon transition, and a second laser, polarized parallel to the applied field, excites continuum states which rapidly ionize. After passing through the second field plate, ions are detected by a channel electron multiplier. The error in scanning the laser to maintain constant scaled energy is $0.002 \mathrm{~cm}^{-1}$, the fractional error in the electric field is $0.3 \%$, and the fractional error in the scaled energy is $0.15 \%$.

We performed scaled-energy spectroscopy on $m=0$ states of lithium for scaled energies between $\epsilon=-2.1$ and $\epsilon=-0.37$. Recurrence spectra were obtained by Fourier transforming the photoabsorption cross section. A panoramic map of the recurrence spectra is shown in Fig. 3. We observe small recurrences corresponding to repetitions of the parallel orbit, and large recurrences near bifurcations. The large bifurcation peaks are labeled with the fractions $m / l$, used to designate the newly created orbits. These orbits are displayed in Fig. 3.

Our experiments and theory are consistent. As the scaled energy approaches the bifurcation from above,

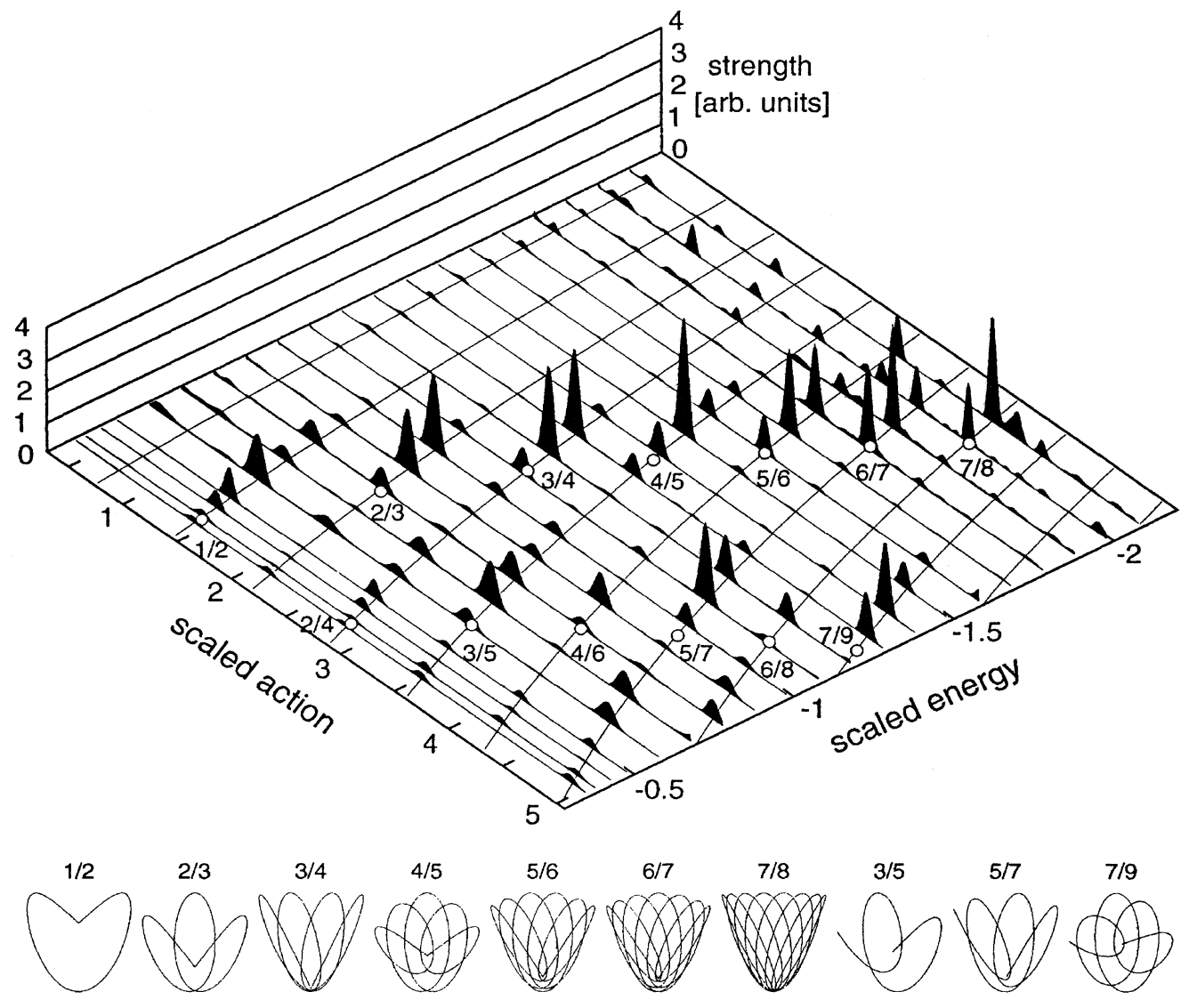

FIG. 3. The curves in the horizontal plane represent the scaled action of the parallel orbit and its repetitions as a function of scaled energy. Locations of bifurcations are marked with small open circles. New orbits created in bifurcations have almost the same action as the corresponding return of the parallel orbit. Measured recurrence strengths are shown in the $z$ direction. Recurrences are especially strong at scaled energies slightly lower than bifurcations. Orbits created by bifurcation of the parallel orbit are shown along the bottom. The $2 / 4,4 / 6$, and $6 / 8$ orbits are repetitions of the $1 / 2,2 / 3$, and $3 / 4$ orbits, respectively, so their shapes are identical. 
the recurrence strength increases significantly. The uncertainty principle allows the quantum system to "see" the bifurcation before it occurs classically [15]. As the scaled energy passes through the bifurcation the recurrence strength increases rapidly, achieving its maximum value noticeably after the bifurcation.

To test the modified closed-orbit theory near bifurcations, we measured scaled-energy spectra near the $1 / 2$ and $2 / 4$ bifurcations in small steps of $\epsilon$. The results are displayed in Fig. 4. The stick spectra represent the computed recurrence strengths, and the solid lines are convolutions of the stick spectra to account for the experimental resolution of the recurrence spectra. The dotted lines are measured recurrence spectra. The $1 / 2$ and $2 / 4$ bifurcations affect peaks 2 and 4 , respectively. Their bifurcation energy is $\epsilon=-0.4$. There are two orbits underneath peaks 2 and 4 , but they are not distinguishable because their actions are nearly equal. Multiple orbits are visible in the stick spectra under peaks 3 and 5 . These orbits were created by the $1 / 3$ and $2 / 5$ bifurcations, respectively. Note that the contributions of two orbits to a single recurrence peak add
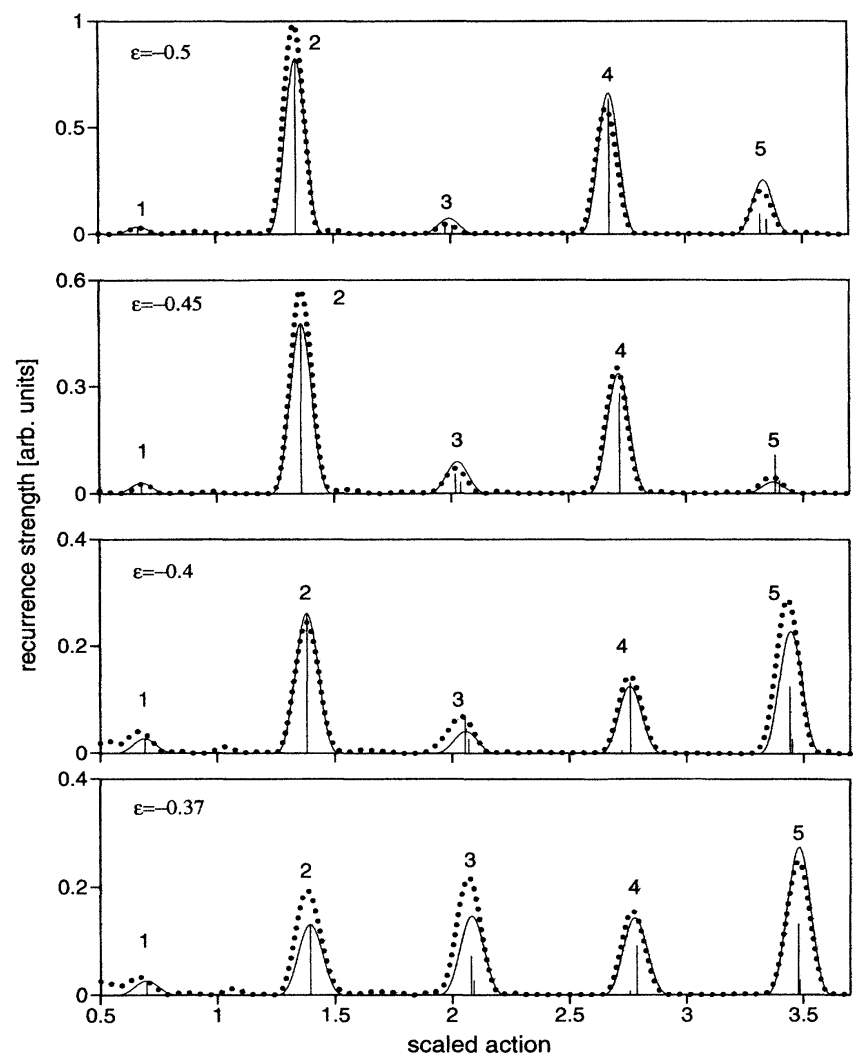

FIG. 4. Recurrence strength near the $1 / 2$ bifurcation. $\epsilon=$ -0.4 is the bifurcation energy. Stick spectra: theory. Solid line: theory smoothed for comparison with experiment. Dotted line: experiment. coherently. For example, peak 5 at $\epsilon=-0.45$ is smaller than the stick spectra, indicating a destructive interference. At $\epsilon=-0.5$, peak 5 displays constructive interference.

These results provide a clear picture of the role of bifurcations in the observed spectrum and detailed confirmation of the closed-orbit theory. They illustrate the fundamental process by which new orbits are created as the energy is decreased and a simple continuum spectrum becomes complex and discrete. The bifurcation process studied here is generic to single-electron atoms. Similar bifurcations occur in diamagnetic hydrogen and lead to the proliferation of recurrences that characterizes classical chaos. Consequently, this study of bifurcations illuminates a fundamental process that connects classical and quantum descriptions of both regular and chaotic systems.

The work at M.I.T. is supported by NSF Grant No. 9221489-PHY and ONR Grant No. N0001490-J-1322. The work at William and Mary is supported by the Jeffress Foundation, ONR, and NSF.

[1] R. R. Freeman, N.P. Economu, G. C. Bjorkland, and K. T. Lu, Phys. Rev. Lett. 41, 1463 (1978); T. S. Luk, L. DiMauro, T. Bergeman, and H. Metcalf, ibid. 47, 83 (1981).

[2] E. Luc-Koenig and A. Bachelier, J. Phys. B 13, 1769 (1980); D. Harmin, Phys. Rev. A 26, 2656 (1982).

[3] M.L. Du and J. B. Delos, Phys. Rev. A 38, 1896 (1988).

[4] J. Gao and J. B. Delos, Phys. Rev. A 49, 869 (1994).

[5] M.C. Gutzwiller, Chaos in Classical and Quantum Mechanics (Springer-Verlag, Berlin, 1990).

[6] J. Parker and C. R. Stroud, Jr., Phys. Rev. Lett. 56, 716 (1986)

[7] U. Eichmann, K. Richter, D. Wintgen, and W. Sandner, Phys. Rev. Lett. 61, 2438 (1988).

[8] A. Holle, J. Main, G. Wiebusch, H. Rottke, and K. H. Welge, Phys. Rev. Lett. 61, 161 (1988).

[9] M. Courtney, H. Jiao, N. Spellmeyer, and D. Kleppner, Phys. Rev. Lett. 73, 1340 (1994).

[10] J. Gao, J. B. Delos, and M. Baruch, Phys. Rev. A 46, 1449 (1992).

[11] J. Main, G. Wiebusch, K. Welge, J. Shaw, and J. B. Delos, Phys. Rev. A 49, 847 (1994).

[12] J. N. L. Conner, Mol. Phys. 31, 33 (1976); J. N. L. Conner and D. Farrelly, J. Chem. Phys. 75, 2831 (1981).

[13] S.P. Parker, Optics Source Book (McGraw Hill, New York, 1988), p. 17; M. Born and E. Wolf, Principles of Optics (Pergamon, New York, 1989), 6th ed.

[14] This results from the fact that the positive and negative $z$ axes are perpendicular in semiparabolic coordinates. A four-dimensional space is needed to describe the symmetry in semiparabolic coordinates.

[15] M. Kus, F. Haake, and D. Delande, Phys. Rev. Lett. 71, 2167 (1993). 